\title{
A comparison of drift wave stability in stellarator and tokamak geometry
}

\author{
J. Anderson, ${ }^{\text {a) }}$ T. Rafiq, M. Nadeem, and M. Persson ${ }^{\text {b) }}$ \\ Department of Electromagnetics, EURATOM-VR Association, Chalmers University of Technology, \\ S-417296 Göteborg, Sweden
}

(Received 2 October 2001; accepted 4 January 2002)

\begin{abstract}
The influence of plasma geometry on the linear stability of electrostatic ion-temperature-gradient driven drift modes (ITG or $\eta_{i}=L_{n} / L_{T i}$ modes) is investigated. An advanced fluid model is used for the ions together with Boltzmann distributed electrons. The derived eigenvalue equation is solved numerically. A comparison is made between an H-1NF [Fusion Technol. 17, 123 (1990)] like stellarator equilibrium, a numerical tokamak equilibrium and the analytical $\hat{s}-\alpha$ equilibrium. The numerical and the analytical tokamak are found to be in good agreement in the low inverse aspect ratio limit. The growth rates of the tokamak and stellarator are comparable whereas the modulus of the real frequency is substantially larger in the stellarator. The threshold in $\eta_{i}$ for the stellarator is found to be somewhat larger. In addition, a stronger stabilization of the ITG mode growth is found for large $\epsilon_{n}\left(=L_{n} / R\right)$ in the stellarator case. (C) 2002 American Institute of Physics.
\end{abstract}

[DOI: $10.1063 / 1.1466820]$

\section{INTRODUCTION}

One of the main unsolved questions in fusion research is to understand the anomalously high cross field particle and energy transport in magnetically confined plasmas. ${ }^{1,2}$ In recent years, substantial efforts have been made towards a deeper understanding of these issues.

One of the most important problems remaining is the effects of three-dimensional (3D) geometry of stellarators and tokamaks, since most of the previous studies have used simplified magnetic field configurations. The geometry affects the magnetic drift frequency, and the perpendicular and parallel space variation, and thus change the regions of favorable and unfavorable curvature.

Among the drift waves, the ion-temperature-gradient (ITG or $\eta_{i}=L_{n} / L_{T i}$ ) driven mode ${ }^{3-6}$ is one of the main candidates for explaining the anomalous transport in the core plasma.

Previous work on stability of $\eta_{i}$-modes include some work on simplified tokamak equilibria ${ }^{7,8}$ and, to a more limited extent, in full stellarator equilibria. ${ }^{9}$ In recent investigations, a full 3D tokamak geometry ${ }^{10}$ was used to calculate the $\eta_{i}$-mode stability.

With inclusion of the nonlinear terms, the model used here has been successful in reproducing experimental ${ }^{11}$ and nonlinear gyrokinetic results. ${ }^{12}$ The drift wave sensitivity to effects of geometry is strongly dependent on the fluid closure and thus it is essential to study the effects of geometry with an advanced fluid model. ${ }^{13}$

In the present paper a comparative investigation of the linear $\eta_{i}$-mode stability on geometry is displayed for three different cases, namely the $\mathrm{H}-1 \mathrm{NF}^{14}$ stellarator, a numerical circular 3D tokamak and an analytical $\hat{s}-\alpha$ equilibrium

\footnotetext{
${ }^{a)}$ Electronic mail: elfja@elmagn.chalmers.se

${ }^{b)}$ Electronic mail: elfmp@elmagn.chalmers.se
}

model. The numerical equilibria employed are generated by the VMEC code. ${ }^{15}$

In this analysis, good agreement is found in comparisons of the analytical and the numerical tokamak results for small and intermediate radial positions. These results are contrasted to the comparisons of the geometry dependent quantities, the magnetic drift frequency and the perpendicular wave vector. The growth rates in the tokamak and stellarator are comparable, whereas the modulus of the real frequency is substantially larger for the stellarator, which is caused by the stronger curvature for the stellarator. The threshold in $\eta_{i}$ for the stellarator is somewhat larger. In addition, a stronger stabilization of the ITG mode growth is found for large $\epsilon_{n}$ in the stellarator case, this is due to the negative local magnetic shear in the stellarator.

It is often assumed that there is a relationship between the linear growth rate and the nonlinear turbulence level. In Ref. 12 there are indications of limitations for this assumption, due to the effects of zonal flows. Zonal flows, radially localized and strongly sheared flows in the poloidal direction are commonly believed to be responsible for the turbulence suppression and enhancement of confinement in toroidal devices. The results reported in Ref. 12 suggests that zonal flows may cause a nonlinear up-shift in the critical temperature gradient needed to obtain transport for longer time scales. This is, however, out of the scope of the present paper.

The remainder of the paper is structured in the following way. In Sec. II the physical model and the equilibria are presented. The results and a discussion thereof is presented in Sec. III. Finally, we summarize the work in Sec. IV.

\section{DRIFT WAVE MODEL AND MAGNETIC FIELD CONFIGURATION}

For the ions, we employ an advanced fluid model, ${ }^{7,8,16,17}$ whereas the electrons are assumed to be Boltzmann distributed. 
The model equations are solved using numerical equilibria and a generalized $\hat{s}-\alpha$ model equilibrium, which allows for the choosing of the global shear $\hat{s}$ and safety factor $q$ as free parameters.

First we consider the fluid model consisting of the ion continuity, the parallel momentum and the ion energy equation in the electrostatic limit,

$\frac{\partial n_{i}}{\partial t}+\nabla \cdot\left(n_{i} \vec{v}_{E}+n_{i} \vec{v}_{\star i}\right)+\nabla \cdot\left(n_{i} \vec{v}_{p i}+n_{i} \vec{v}_{\pi i}\right)+\nabla \cdot\left(n_{i} \vec{v}_{\| i}\right)=0$,

$m_{i} n_{i} \frac{\partial \vec{v}_{\| i}}{\partial t}+\nabla_{\|} p_{i}+n_{i} e \nabla_{\|} \phi=0$

$\frac{3}{2} n_{i} \frac{d T_{i}}{d t}+n_{i} T_{i} \nabla \cdot \vec{v}_{i}+\nabla \cdot \vec{q}_{i}=0$.

Here, $n_{i}$ is the ion particle density, $p_{i}=n_{i} T_{i}$ is the ion pressure, $T_{i}$ is the ion temperature, $\vec{v}_{\| i}$ is the parallel ion velocity, $\phi$ is the electrostatic potential, and $m_{i}$ is the ion mass. The convective derivative is defined as $d / d t=(\partial / \partial t)+\left(\vec{v}_{E}\right.$ $\left.+\vec{v}_{\star i}\right) \cdot \nabla$. The diamagnetic drift and $\vec{E} \times \vec{B}$ drifts are defined as

$$
\begin{aligned}
& \vec{v}_{\star i}=\frac{c}{e n_{i} B}\left(e_{\|} \times \nabla p_{i}\right), \\
& \vec{v}_{E}=\frac{c}{B}\left(e_{\|} \times \nabla \phi\right),
\end{aligned}
$$

where $e$ is the charge, $c$ is the speed of light, and $e_{\|}$ $=\vec{B} /|\vec{B}|$ is a unit vector along the magnetic field. The drift $\vec{v}_{\pi i}$ is the stress tensor drift, cf. p. 20, Ref. 16, and the polarization drift $v_{p_{i}}$ is defined as

$$
\vec{v}_{p_{i}}=\frac{1}{\Omega_{c i}}\left(\frac{\partial}{\partial t}+\vec{v}_{i} \cdot \nabla\right)\left(e_{\|} \times \vec{v}_{i}\right),
$$

where $\vec{v}_{i}$ is the total ion drift velocity and the cyclotron frequency is given by $\Omega_{c i}=e B / m_{i} c$. Here we express the divergence as

$$
\nabla \cdot\left(n_{i} \vec{v}_{p i}+n_{i} \vec{v}_{\pi i}\right)=\nabla \cdot\left(\frac{n_{i}}{\Omega_{c i}} \frac{\partial}{\partial t}\left(e_{\|} \times \vec{v}_{i}\right)\right),
$$

where we substitute $\vec{v}_{E}$ and $\vec{v}_{\star i}$ for $\vec{v}_{i}$. The part due to $\vec{v}_{E}$ corresponds to the polarization drift velocity, and the part due to $\vec{v}_{\star i}$ is the lowest order finite Larmor radius (FLR) term. We then obtain

$$
\nabla \cdot\left(n_{i} \vec{v}_{p i}+n_{i} \vec{v}_{\pi i}\right)=-n_{i} \rho_{s}^{2} \frac{\partial}{\partial t} \Delta \frac{e \phi}{T_{e}}-\frac{\rho_{s}^{2}}{T_{e}} \frac{\partial}{\partial t} \Delta \delta p_{i},
$$

where $\rho_{s}=c_{s} / \Omega_{c i}$ and $c_{s}=\sqrt{T_{e} / m_{i}}$ and $\Delta$ is the Laplacian. The diamagnetic ion heat flux is given by

$$
\vec{q}_{i}=\vec{q}_{\star i}=\frac{5}{2} \frac{c T_{i} n_{i}}{e B} e_{\|} \times \nabla T_{i}
$$

The ion temperature fluctuations equations couple to Eqs. (1) and (2) through

$$
\nabla \cdot\left(n_{j} \vec{v}_{\star j}\right)=\frac{1}{T_{j}} \vec{v}_{D j} \cdot \nabla \delta P_{j},
$$

where $\delta P_{j}(j=i, e)$ is the perturbed pressure and $\vec{v}_{D j}$ is the magnetic drift velocity at the thermal speed, i.e.,

$$
\vec{v}_{D i}=\frac{T_{i}}{m_{i} \Omega_{c i}}\left[\frac{e_{\|} \times \nabla B}{B}+e_{\|} \times \vec{\kappa}\right],
$$

where $\vec{\kappa}=\left(e_{\|} \cdot \nabla\right) e_{\|}$is the curvature vector. Additional curvature relations arise in Eqs. (1) and (2) as

$$
\begin{aligned}
& \nabla \cdot \vec{q}_{\star i}=-\frac{5}{2} n_{i} \vec{v}_{\star i} \cdot \nabla T+\frac{5}{2} n_{i} \vec{v}_{D i} \cdot \nabla T, \\
& \nabla \cdot \vec{v}_{E}=\frac{q_{j}}{T_{j}} \vec{v}_{D j} \cdot \nabla \phi .
\end{aligned}
$$

Assuming a perturbation of the form $e^{-i \omega t}$ and using the continuity equation for $\nabla \cdot \vec{v}_{i}$ in the energy equation, the convective diamagnetic effects cancel out and the energy equation becomes

$$
\frac{\delta T_{i}}{T_{i}}=\frac{\omega}{\omega-\frac{5}{3} \omega_{D_{i}}}\left(\frac{2}{3} \frac{\delta n_{i}}{n_{0}}-\frac{\omega_{\star e}}{\omega}\left(\frac{2}{3}-\eta_{i}\right) \frac{e \phi}{T_{e}}\right) .
$$

The parallel ion equation (2) gives

$$
\vec{v}_{\| i}=-i \frac{c_{s}^{2}}{\omega} e_{\|} \cdot \nabla\left(\frac{e \phi}{T_{e}}+\frac{1}{\tau} \frac{\delta p_{i}}{p_{i}}\right) .
$$

Here we have $\tau=T_{e} / T_{i}$. Substituting (13) and (14) into (1) and using quasineutrality and Boltzmann distributed electrons $\delta n_{i} / n_{0}=\delta n_{e} / n_{0}=e \phi / T_{e}$, we arrive at the eigenvalue equation

$$
\begin{aligned}
c_{s}^{2}\left(e_{\|} \cdot \nabla\right)^{2}\left(1+\frac{1}{\tau}+\hat{F}\right) \frac{e \phi}{T_{e}} \\
=-\omega\left(\omega-\omega_{\star e}\right) \frac{e \phi}{T_{e}}+\omega\left(\tau \omega_{D_{i}}-\omega k_{\perp}^{2} \rho_{s}^{2}\right) \\
\times\left(1+\frac{1}{\tau}+\hat{F}\right) \frac{e \phi}{T_{e}},
\end{aligned}
$$

where

$$
\hat{F}=\frac{\omega}{\omega-\frac{5}{3} \omega_{D_{i}}}\left(\frac{2}{3 \tau}+\frac{\omega_{\star i}}{\omega}\left(\frac{2}{3}-\eta_{i}\right)\right) .
$$

Employing the standard high- $n$ ballooning mode formalism, ${ }^{18}$ we obtain a second order eigenvalue equation for the potential $\phi$ in the extended ballooning mode coordinate $\xi$,

$$
\left(e_{\|} \cdot \nabla\right)^{2} \Phi=-h\left(\left(1-\frac{1}{\Omega}\right) A-\frac{2 \tau \omega_{D_{i}}}{\omega}+k_{\perp}^{2} \rho_{s}^{2}\right) \Phi,
$$

where $\Omega=\omega / \omega_{\star e}$ and we have

$$
\Phi=\left(1+\frac{1}{\tau}+\hat{F}\right) \frac{e \phi}{T_{e}},
$$




$$
\begin{aligned}
& A=\frac{1-\frac{10}{3} \frac{\omega_{D_{i}}}{\omega}}{F-2 \beta \tau \frac{\omega_{D_{i}}}{\omega}}, \\
& F=1+\frac{1}{\Omega \tau}\left(1+\eta_{i}\right)+\frac{5}{3 \tau}\left(1-\frac{1}{\Omega}\right), \\
& \beta=\frac{5}{3 \tau}\left(1+\frac{1}{\tau}\right), \\
& h=\frac{q^{2} k_{\theta}^{2} \rho_{s}^{2} \tau \Omega^{2}}{\epsilon_{n}^{2}} .
\end{aligned}
$$

Here, $\epsilon_{n}=L_{n} / R$ and $q$ is the safety factor, $\hat{s}=d \ln q / d \ln r$ is the magnetic shear. The geometric effects enter through the magnetic drift frequency $\omega_{D}$ and the parallel derivative in the second order differential eigenvalue equation and through $k_{\perp}^{2}$.

The numerical equilibria are calculated using the VMEC code ${ }^{15}$ and are then mapped to the Boozer coordinate system $(s, \theta, \xi),{ }^{19,20}$ where $s=2 \pi \psi / \psi_{p}$ is the normalized flux (radial) coordinate and $\theta, \xi$ are the generalized poloidal and toroidal angles, respectively. Here $\psi_{p}=\pi B_{0} \bar{a}^{2} / q$ is the total poloidal magnetic flux and $2 \pi \psi$ is the poloidal magnetic flux, where $B_{0}$ is the magnetic field at the axis, $\bar{a}$ is the minor radius. The Boozer coordinate system is related to the Cartesian coordinate system $(x, y, z)$ by the cylindrical coordinates $(R, \phi, z)$, where $R$ is the major axis and $\phi$ the cylindrical toroidal angle, as

$$
\begin{aligned}
& R=\sum_{m=0}^{n_{p}} \sum_{n=-n_{t}}^{n_{t}} R_{m n}(s) \cos (m \theta+n N \xi), \\
& \phi=\xi-\frac{2 \pi}{N} \sum_{m=0}^{n_{p}} \sum_{n=-n_{t}}^{n_{t}} \phi_{m n}(s) \sin (m \theta+n N \xi), \\
& z=\sum_{m=0}^{n_{p}} \sum_{n=-n_{t}}^{n_{t}} z_{m n}(s) \sin (m \theta+n N \xi) .
\end{aligned}
$$

Here $n_{p}$ and $n_{t}$ are the maximum poloidal and toroidal Fourier components, respectively, and $N$ is the number of field periods in the device studied. The Fourier components $R_{m n}, \phi_{m n}, z_{m n}$ and the rotational transform $\iota=2 \pi / q$ are determined by the VMEC code.

The position vector $\vec{r}$ of any point $(s, \theta, \xi)$ at a magnetic surface $s$ in the Cartesian coordinate system is $\vec{r}$ $=(R \cos \phi, R \sin \phi, z)$ with the covariant basis vectors in the Boozer system $\vec{e}_{i}=\partial \vec{r} / \partial x^{i}$, where $x^{i}=\{s, \theta, \xi\}$ and the contravariant components are calculated as $\nabla x^{i}=\vec{e}_{j} \times \vec{e}_{k} / J$. Here

$$
J=\vec{e}_{s} \cdot \vec{e}_{\theta} \times \vec{e}_{\xi}=\frac{\bar{R} \dot{\psi}}{B^{2}}\left(B_{\theta}+q B_{\xi}\right)
$$

is the Jacobian, where $\vec{B}=\nabla \alpha \times \nabla \psi=\dot{\psi} \nabla \alpha \times \nabla s, \quad \dot{\psi}$ $=d \psi / d s=B_{0} \bar{a} / 2 q$, and $\alpha=\xi-q \theta$. The magnetic field $\vec{B}$ fulfills $\nabla \cdot \vec{B}=0$ and $\vec{B} \cdot \nabla \psi=0=\vec{B} \cdot \nabla \alpha$ which is equivalent to the fact that $\psi$ and $\alpha$ are stream functions of the magnetic field.

Now employing the formulas for contra and covariant basis vectors, the magnetic field can be expressed as $\vec{B}$ $=\dot{\psi} / J\left(\vec{e}_{\theta}+q \vec{e}_{\xi}\right)$ and the operator

$$
\vec{e}_{\|} \cdot \nabla=\frac{\dot{\psi}}{J B}\left(\frac{\partial}{\partial \theta}+q \frac{\partial}{\partial \xi}\right)=\frac{\dot{\psi} q}{J B} \frac{d}{d \xi}
$$

and the field line curvature vector

$$
\begin{aligned}
\vec{\kappa}=\vec{e}_{\|} \cdot \nabla \vec{e}_{\|}= & q\left(\frac{\dot{\psi}}{J B}\right)^{2}\left(\frac{d}{d \xi}\left(\vec{e}_{\theta}+q \vec{e}_{\xi}\right)\right. \\
& \left.-\frac{1}{2} \frac{d \ln J}{d \xi}\left(\vec{e}_{\theta}+q \vec{e}_{\xi}\right)\right) .
\end{aligned}
$$

The eigenmode equation is now in the Boozer coordinate system,

$$
\begin{aligned}
\frac{d^{2}}{d \xi^{2}} \Phi= & \left(\frac{2 \chi J B}{\bar{a} \epsilon_{n} q \bar{R} \dot{\psi}}\right)^{2}\left(\left(H^{-1}-\frac{\bar{a} \epsilon_{n} \Omega_{D}}{2}\right) \Omega\right. \\
& \left.-\left(H^{-1}+\left(\frac{\chi B_{0}}{B}\right)^{2} \hat{k}_{\perp}^{2}\right)\right) \Phi,
\end{aligned}
$$

where

$$
\begin{aligned}
\Phi & =H \frac{e \phi}{T_{e}}, \\
H & =1+\tau^{-1}+\frac{\tau^{-1}\left(\frac{2}{3} \Omega+\eta_{i}-\frac{2}{3}\right)}{\Omega+\frac{5}{6 \tau} \bar{a} \epsilon_{n} \Omega_{D}}, \\
\chi= & \epsilon^{-1} \frac{q \rho_{s}}{\bar{a}} \frac{\partial S}{\partial \alpha}, \\
\omega_{D_{i}}= & \omega_{D_{i}}(s, \alpha, \xi)=\frac{B_{0} \bar{R}}{B^{2}} \vec{k}_{\perp} \cdot(\vec{B} \times(\kappa+\nabla \ln B)), \\
\vec{k}_{\perp}= & \vec{k}_{\perp}\left(s, \alpha, \xi ; \theta_{k}\right) \\
& =\frac{\bar{a}}{q}\left(\nabla \xi-q \nabla \theta-\left(\frac{\xi-\xi_{0}}{q}-\theta_{k}\right) \frac{d q}{d \psi} \nabla \psi\right),
\end{aligned}
$$

where $\epsilon$ is the WKB expansion parameter and $S$ is the eikonal in the standard ballooning mode formalism. This definition of $\theta_{k}$ differs slightly from that commonly used in such a way that it gives the same $k_{\perp}$ for different matching points. Using the analytical $\hat{s}-\alpha$ equilibrium model, explicit expressions for the magnetic drift frequency and perpendicular space variation are found

$$
\begin{aligned}
& \omega_{D}=2 \omega_{\star} \epsilon_{n}\left(\cos \xi+\hat{s} \xi \sin \xi-\frac{\bar{a}}{\bar{R}}\right), \\
& k_{\perp}^{2} \rho_{s}^{2}=b \tau\left(1+\hat{s}^{2} \xi^{2}\right) .
\end{aligned}
$$




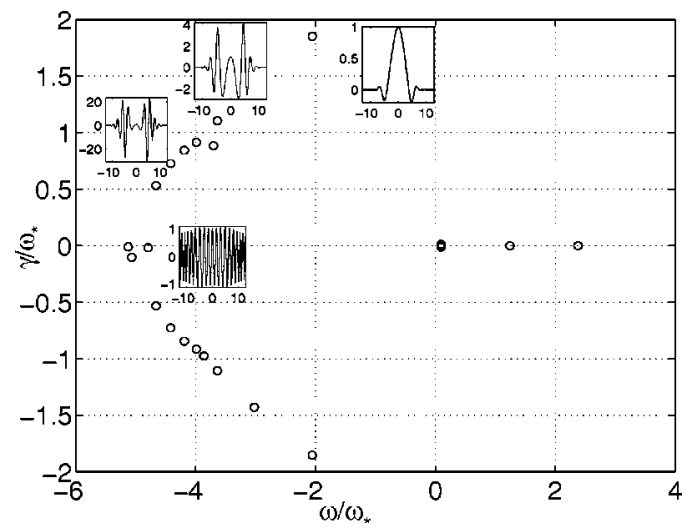

FIG. 1. The tokamak spectrum [the growth rate $\gamma$ vs the real frequency $\omega_{r}$ (normalized to the electron diamagnetic drift frequency)] of the drift wave equation for $s=0.4, b=0.1, \theta_{k}=0.0, \epsilon_{n}=0.5, \eta_{i}=8$, and $\tau=1$. The corresponding eigenfunctions along $L / R q$ are shown as insets.

Here $b=k_{\theta}^{2} \rho_{S}^{2}$ and the global magnetic shear is calculated from the numerical equilibrium as $\hat{s}=(1 / q)(\partial q / \partial s)$ $\times(\partial s / \partial r)$, cf. Ref. 21 .

\section{RESULTS AND DISCUSSION}

The solutions for the eigenmode equation [Eq. (29)] are found by a standard numerical shooting technique, making use of the WKB-type boundary conditions for large $\xi$ as in Refs. 8 and 22. These studies are carried out on the $\mathrm{H}-1 \mathrm{NF}$
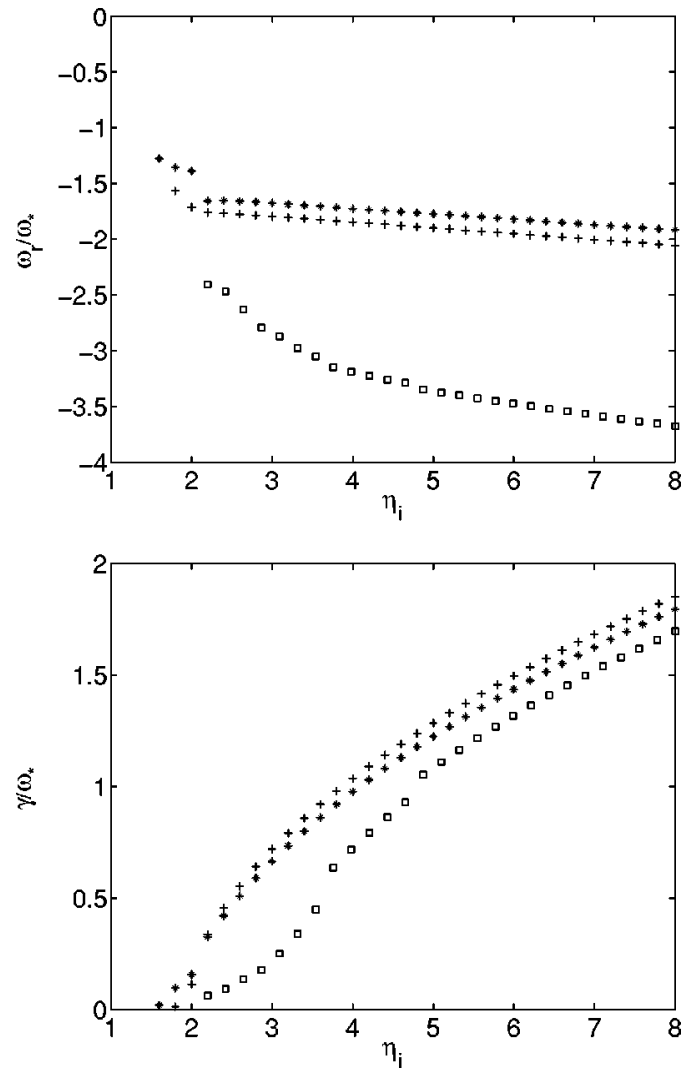

FIG. 2. The frequency (top) and the growth rate (bottom) (normalized to the electron diamagnetic drift frequency) vs $\eta_{i}$ (* representing the analytical tokamak, + the numerical tokamak, and the squares the stellarator) for $s$ $=0.4, b=0.1, \theta_{k}=0.0, \epsilon_{n}=0.5$, and $\tau=1$.
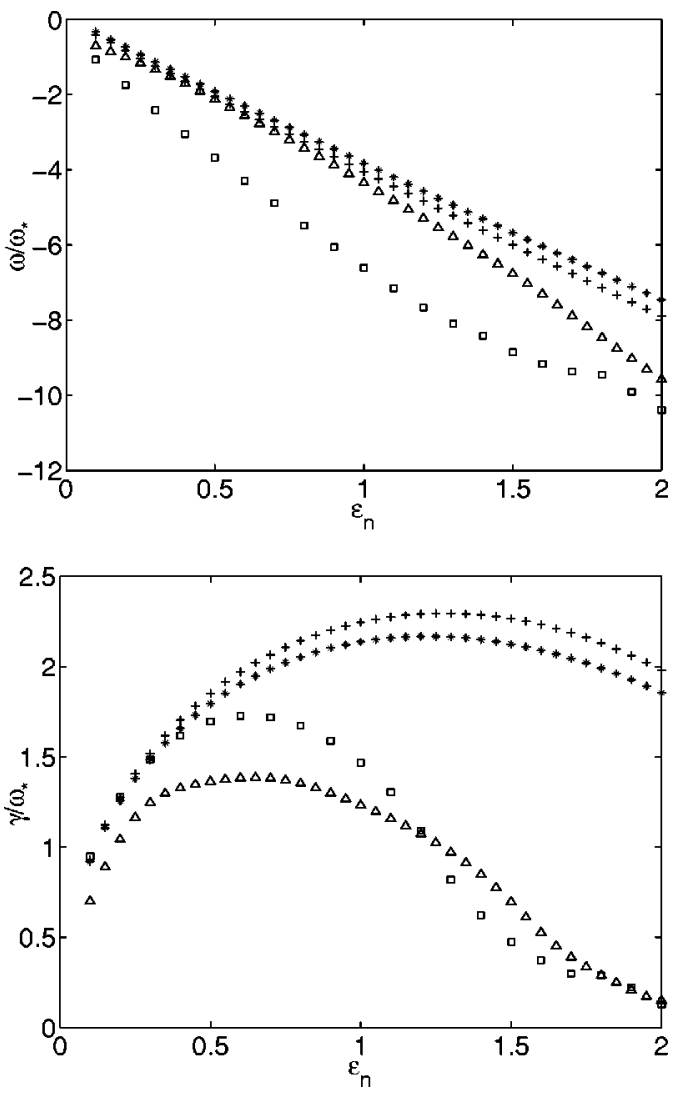

FIG. 3. The frequency (top) and the growth rate (bottom) (normalized to the electron diamagnetic drift frequency) vs $\epsilon_{n}[*(\hat{s}=0.459)$ and triangles $(\hat{s}$ $=-0.459)$ representing the analytical tokamak, + the numerical tokamak, and the squares the stellarator] for $s=0.4, b=0.1, \theta_{k}=0.0, \eta_{i}=8$, and $\tau$ $=1$.

stellarator with $n_{p}=13, n_{t}=27$ and with field configuration having a three-fold toroidal symmetry $(N=3)$ and a numerical tokamak equilibrium having an aspect ratio $(\bar{R} / \bar{a})=10$, which is also compared to the analytical $\hat{s}-\alpha$ model equilibrium.

The ITG mode spectrum (normalized to the electron diamagnetic frequency) is shown in Fig. 1 for the Numerical Tokamak for $s=0.4, b=0.1, \theta_{k}=0.0, \epsilon_{n}=0.5, \eta_{i}=8$, and $\tau=1$. The eigenfunctions along the field line are displayed as insets for some of the eigenfrequencies. It is found that the mode growth of the stellarator and the tokamak are comparable, whereas the modulus of the real frequency is substantially lower in the tokamak than the stellarator, cf. Fig. 1 in Ref. 9. In Ref. 16, the real frequency and the growth rate are calculated analytically. Neglecting the effects of FLR and letting $\tau=1$, the real part of the frequency can be written as

$$
\frac{\omega_{r}}{\omega_{\star}}=-\frac{1}{2}\left[-1+\frac{13}{3}\left|\omega_{D} / \omega_{\star}\right|\right] .
$$

If, as in the stellarator case, the curvature $\left(\left|\omega_{D} / \omega_{\star}\right|(\xi=0)\right.$ $\approx 1.37)$ is increased [compared to the analytical tokamak $\left(\left|\omega_{D} / \omega_{\star}\right|(\xi=0)=1.0\right)$ which also holds for the numerical tokamak] then the real frequency is increased. Qualitatively, this explains the shift in frequency between Fig. 1 in this paper and Fig. 1 of Ref. 9. The eigenfunctions for the most 

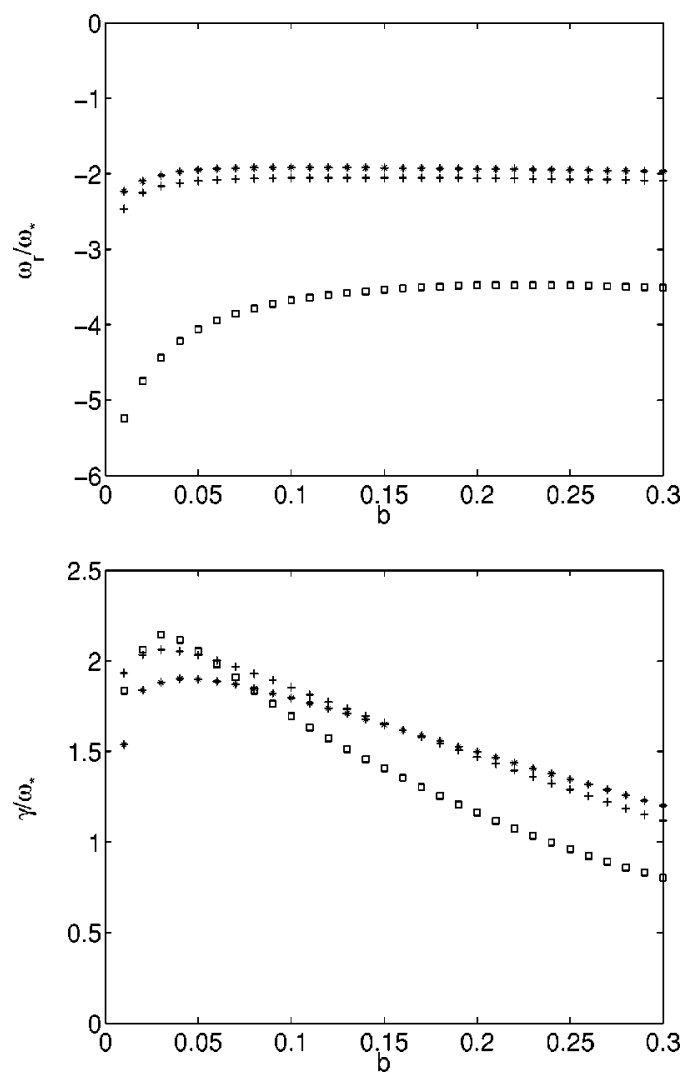

FIG. 4. The frequency (top) and the growth rate (bottom) (normalized to the electron diamagnetic drift frequency) vs $b$ (* representing the analytical tokamak, + the numerical tokamak, and the squares the stellarator) for $s$ $=0.4, \theta_{k}=0.0, \epsilon_{n}=0.5, \eta_{i}=8$, and $\tau=1$.

unstable mode, which is also the most localized mode, are very similar comparing the stellarator and tokamak case.

Figure 2 displays the mode eigenfrequency (both real frequency and growth rate normalized to the electron diamagnetic frequency) as a function $\eta_{i}(*$ representing analytical tokamak, + numerical tokamak and the squares the stellarator) and the other parameters as in Fig. 1. A good agreement in the growth rate above the threshold for the analytical and numerical tokamak is found. However, there is a slight shift in the real frequency. The modulus of the real frequency for the stellarator is substantially larger and the threshold is somewhat larger than in the tokamak case. There are previous results indicating that negative magnetic shear increases the threshold, cf. Fig. 7 of Ref. 8.

In Fig. 3 the $\epsilon_{n}$ dependency of the eigenvalue on both real frequency and growth rate normalized to the electron diamagnetic frequency and the other parameter values as in Fig. 1 are displayed, with the symbol $*$ representing the analytical tokamak, triangles the analytical tokamak with reversed shear $(\hat{s}=-0.459),+$ the numerical tokamak, and the squares the stellarator. Note the stabilization due to compression is recovered for smaller values in the stellarator than in the tokamak geometry. This is due to the fact that the negative magnetic shear in the stellarator tends to decrease the curvature and the mode eigenfunction becomes extended and, thus, the growth rate decreases. Using the analytical model with reversed shear, the stellarator result is repro-
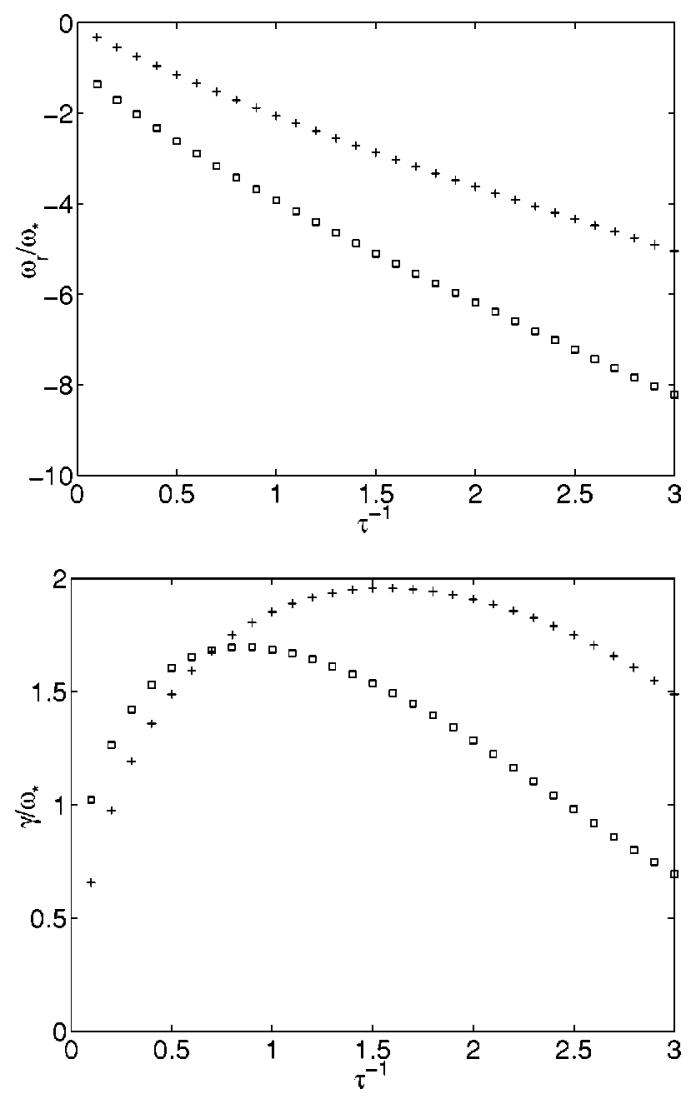

FIG. 5. The frequency (top) and the growth rate (bottom) (normalized to the electron diamagnetic drift frequency) vs $\tau^{-1}$ ( + representing the numerical tokamak and the squares the stellarator) for $s=0.4, b=0.1, \theta_{k}=0.0, \epsilon_{n}$ $=0.5$, and $\eta_{i}=8$.

duced. Moreover, there is good agreement of the growth rates for all three geometries for small $\epsilon_{n}$.

The effects of finite Larmor radius are presented in Fig. 4. Here $b$, defined as

$$
b=k_{\theta}^{2} \rho_{s}^{2}=\left.\left(\epsilon^{-1} \frac{\partial S}{\partial \alpha}\right) \nabla \alpha \cdot \nabla \alpha\right|_{\xi=0},
$$

is the local value of the normalized perpendicular wave number in the magnetic surface. The eigenfrequency, with both real frequency and growth rate normalized to the electron diamagnetic frequency as a function of $b$ is shown and the other parameters are as in Fig. 1. Here the symbol * represents the analytical tokamak, + the numerical tokamak, and the squares the stellarator. Comparing the three different geometries, there are no large shifts in the peak value of the spectrum, whereas in the stellarator case there is stronger stabilization of the growth rate as $b$ increases.

Considering temperature ratios in terms of $\tau^{-1}$ $=T_{i} / T_{e}$, the dependency on the eigenfrequency is normalized to the electron diamagnetic frequency and the other parameters as in Fig. 1 is shown in Fig. 5, + representing the numerical tokamak and the squares the stellarator. The stabilization due to large and small $\tau^{-1}$ is found in both the stellarator and the tokamak, however, the stabilization is somewhat stronger for larger $\tau^{-1}$ and somewhat weaker for smaller $\tau^{-1}$, $\mathrm{s}$ in the stellarator case. 

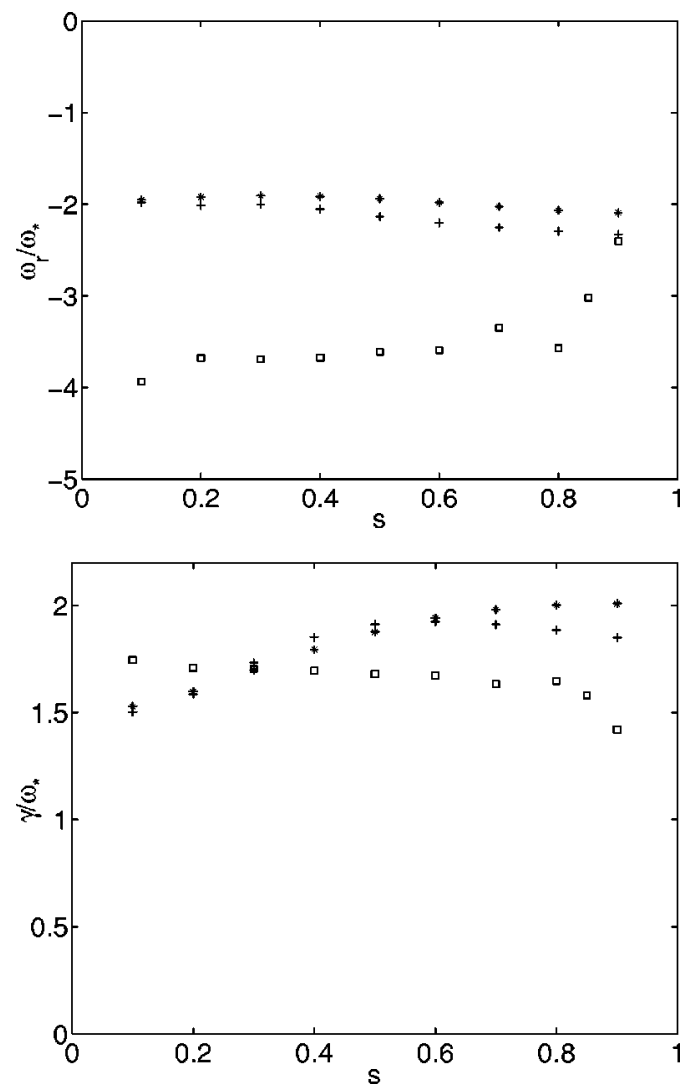

FIG. 6. The frequency (top) and the growth rate (bottom) (normalized to the electron diamagnetic drift frequency) vs $s$ (* representing the analytical tokamak, + the numerical tokamak, and the squares the stellarator) for $b$ $=0.1, \theta_{k}=0.0, \epsilon_{n}=0.5, \eta_{i}=8$, and $\tau=1$.

The variation of the results with aspect ratio and associated equilibrium quantities such as curvature and $\bmod B$ is displayed in Fig. 6, which shows the eigenfrequency as a function of the normalized radial Boozer coordinate $s$. Here the symbol * represents the analytical tokamak, + the numerical tokamak, and squares the stellarator, all other parameters are set as in Fig. 1. It is observed that for small and intermediate $s$, the growth rates agrees fairly well, whereas for large $s$ there is a slight disagreement in the real frequency for the tokamaks. Presumably this is due to the decreasing aspect ratio for larger $s$. For the stellarator, the curvature decreases as $s$ increases and the growth rate and the modulus of the real frequency decrease. Employing Eq. (37), at magnetic surface $s=0.4$ the real frequency for the tokamak is approximately -1.67 and for the stellarator it is -2.47 . If the FLR dependent terms are included, the real frequency will be slightly lower in both cases.

We can understand the differences in the obtained results between stellarators and the tokamaks and the variation with the radial coordinate in terms of the variation of the curvature. We can also understand the different results obtained by using the numerical tokamak and the $\hat{s}-\alpha$ model equilibrium in terms of the different variation of the curvature with respect to the radial coordinate. In Figs. 7(a), 7(b); 8(a), 8(b); 9(a), 9(b) the dashed lines represent the numerical tokamak and the solid lines $(\hat{s}=0.459)$ and dashed-dotted line $(\hat{s}$ $=-0.459)$ the analytical model, where the magnetic drift

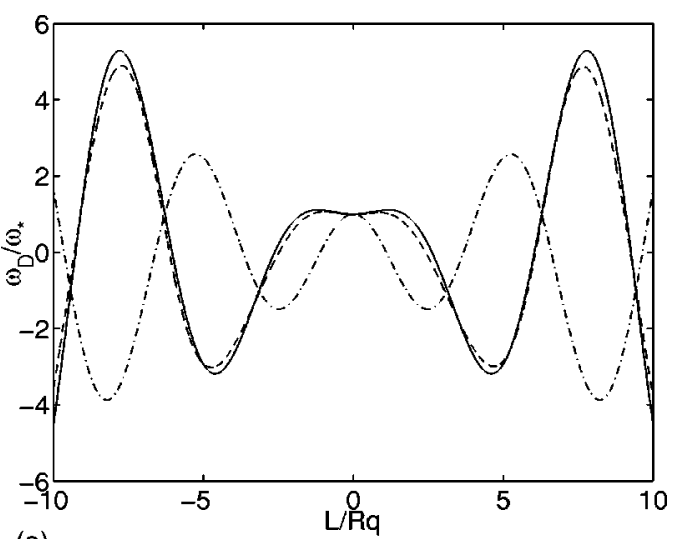

(a)

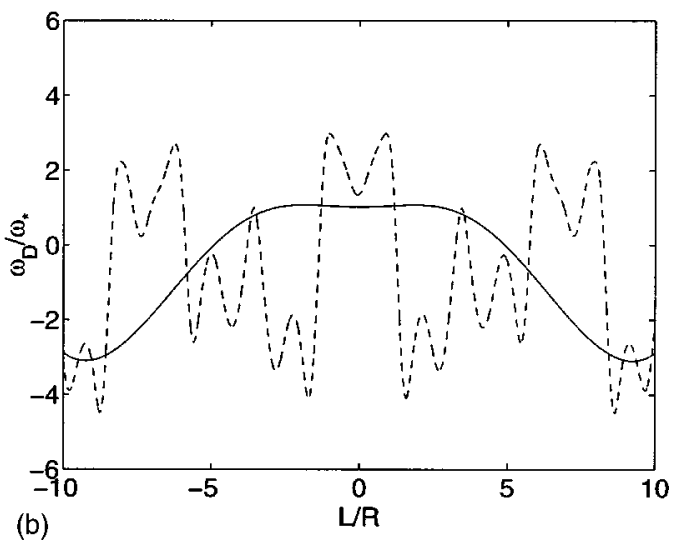

FIG. 7. (a) The function $\omega_{D}$ (normalized to the ion diamagnetic drift frequency) vs $L / R q$ the dashed line representing the numerical tokamak and the solid line $(\hat{s}=0.459)$ and dashed-dotted line $(\hat{s}=-0.459)$ the analytical model for $s=0.4$. (b) The function $\omega_{D}$ (normalized to the ion diamagnetic drift frequency) vs $L / R$ the solid line representing the numerical tokamak and the dashed line the stellarator for $s=0.4$.

frequency is shown for $s=0.4$ [Fig. 7(a)] and $s=0.9$ [Fig. $8(\mathrm{a})]$ as a function of a normalized length $L / R q$. In Figs. $7(\mathrm{~b})$ and $8(\mathrm{~b})$, the magnetic drift frequency as a function of $L / R$ is displayed for the stellarator (dashed line) and the numerical tokamak (solid line). The perpendicular wave vector as a function of $L / R q$ for the two different magnetic surfaces $s=0.4$ [Fig. 9(a)] and $s=0.9$ [Fig. 9(b)] are shown. The corresponding global magnetic shear and safety factor for the two different flux surfaces $s=0.4$ and $s=0.9$ in the tokamak case are $\hat{s} \approx 0.459, q \approx 2.02$ and $\hat{s} \approx 1.10, q \approx 3.10$, respectively. The periodicity of the curvature in Figs. 7(a) and 8(a) is different, since the values of $q$ are different. In the case of higher $q$ value [Fig. 8(a)] the region of bad curvature is significantly larger. When we, in Figs. 7(b) and 8(b), compare the stellarator curvature as a function of the length along the field line, $L / R$, with the corresponding tokamak results, we find that the helical ripples in the stellarator case decrease the connection length between bad and good curvature regions and thus has a stabilizing effect on the modes. In Figs. 7,8 , and 9 good agreement is found for the region where the most localized mode, which has the largest growth rate, is excited, comparing the analytical and numerical tokamak at the $s=0.4$ flux surface, whereas for $s=0.9$ there are differences. In the case of reversed magnetic shear tokamak (dashed-dotted line, $s=-0.459)$ the curvature is signifi- 


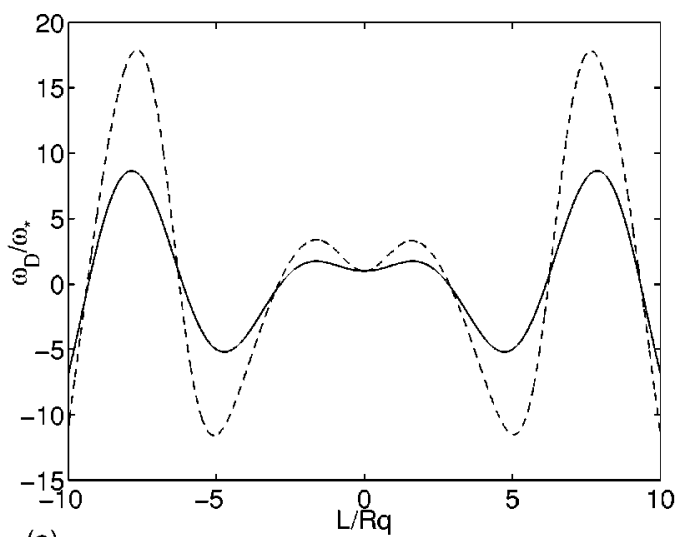

(a)

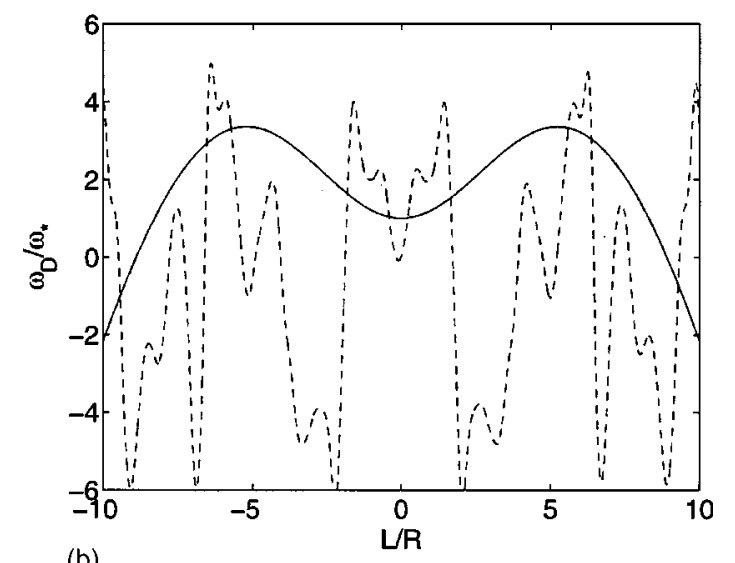

(b)

FIG. 8. (a) The function $\omega_{D}$ (normalized to the ion diamagnetic drift frequency) vs $L / R q$ the dashed line representing the numerical tokamak and the solid line $(\hat{s}=0.459)$ the analytical model for $s=0.9$. (b) The function $\omega_{D}$ (normalized to the ion diamagnetic drift frequency) vs $L / R$ the solid line representing the numerical tokamak and the dashed line the stellarator for $s=0.9$.

cantly reduced and, hence, the eigenfunctions are more extended and the growth rates are lower. In the case of eigenmodes localized in regions with favorable curvature (e.g., the inside of the tokamak) the eigenfunctions tend to become extended and the growth rates are decreased.

\section{SUMMARY}

The effects of 3D geometry in a circular tokamak and the stellarator $\mathrm{H}-1 \mathrm{NF}$ are compared with respect to linear stability of the ion-temperature-gradient ITG mode. The work is based on an advanced fluid model for the ions in the presence of parallel ion momentum effects and the electrons are assumed to be Boltzmannian. The effects of finite $\beta$, trapping, collisions are neglected in order to more easily quantify the effects of 3D geometry. The 3D numerical equilibria are generated by the VMEC code and the analytical equilibrium is the $\hat{s}-\alpha$ model.

An eigenvalue equation was derived using the high- $n$ ballooning mode formalism. The derived eigenvalue equation was solved using a standard shooting technique described in Refs. 8 and 9.

A comparison of the analytical and the numerical tokamak results for small and intermediate radial Boozer coordi-

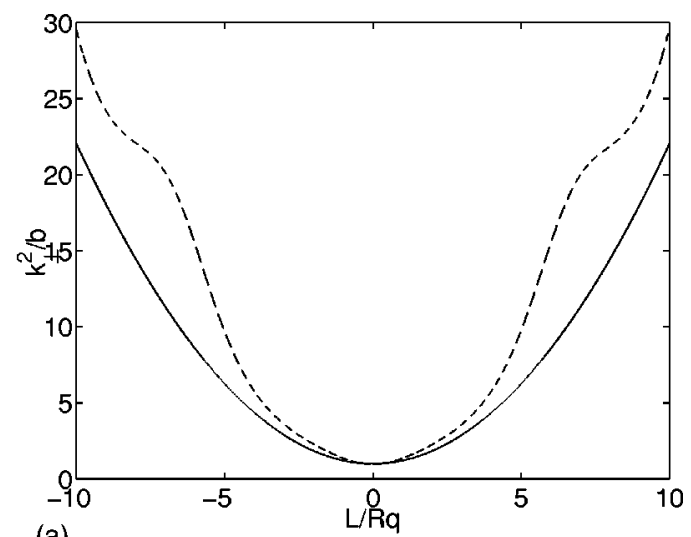

(a)

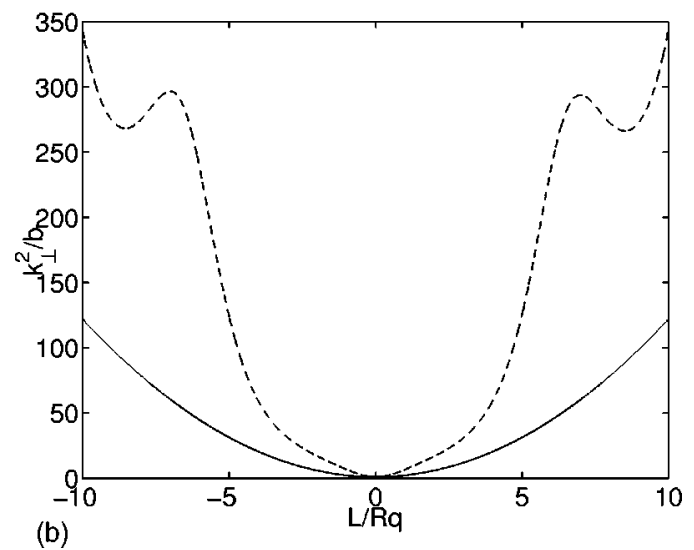

FIG. 9. (a) The function $k_{\perp}^{2}$ [normalized to (b)] vs $L / R q$ the dashed line representing the numerical tokamak and the solid line the analytical model for $s=0.4$. (b) The function $k_{\perp}^{2}$ [normalized to (b)] vs $L / R q$ the dashed line representing the numerical tokamak and the solid line the analytical model for $s=0.9$.

nate $s$ showed good agreement. The differences for larger radii is understood in terms of geometry dependent quantities, the magnetic drift frequency and the perpendicular wave vector. The growth rates in the tokamak and stellarator are comparable, whereas the modulus of the real frequency is substantially larger for the stellarator. The latter can be understood in terms of differences in the geometry resulting in substantially different curvature. The threshold in $\eta_{i}$ for the stellarator is somewhat larger. This is a result of negative magnetic shear, which is also observed in tokamaks with negative shear. The stabilization due to compression is recovered for smaller values of $\epsilon_{n}$ in the stellarator than in the tokamak geometry. This is due to the fact that the negative magnetic shear in the stellarator tends to decrease the curvature and the mode eigenfunction becomes extended and thus the growth rate decreases. This is displayed using the corresponding result for the analytical model with reversed shear.

The effects of noncircular 3D tokamak geometry is important but has been neglected in this work and will be a subject of a future paper. Especially the cross-sectional elongation, since it is known experimentally that the effects of elongation on the energy confinement time is favorable $\tau_{E}$ $\sim \kappa^{0.5} \cdot{ }^{23}$ 


\section{ACKNOWLEDGMENTS}

The authors are grateful to Professor Jan Weiland and Dr. Hans Nordman for discussions and helpful suggestions.

${ }^{1}$ P.C. Liewer, Nucl. Fusion 25, 543 (1985).

${ }^{2}$ F. Wagner and U. Stroth, Plasma Phys. Controlled Fusion 35, 1321 (1993).

${ }^{3}$ L.I. Rudakov and R.Z. Sagdeev, Sov. Phys. Dokl. 6, 415 (1961).

${ }^{4}$ B. Coppi, M.N. Rosenbluth, and R.Z. Sagdeev, Phys. Fluids 10, 582 (1967).

${ }^{5}$ B. Coppi and F. Pegoraro, Nucl. Fusion 17, 969 (1977).

${ }^{6}$ P.N. Guzdar, L. Chen, W.M. Tang et al., Phys. Fluids 26, 673 (1983).

${ }^{7}$ A. Jarmèn, P. Andersson, and J. Weiland, Nucl. Fusion 27, 941 (1987)

${ }^{8}$ S.C. Guo and J. Weiland, Nucl. Fusion 37, 1095 (1997).

${ }^{9}$ T. Rafiq, J. Anderson, M. Nadeem, and M. Persson, Plasma Phys. Controlled Fusion 43, 1363 (2001).

${ }^{10}$ A.J. Redd, A.H. Kritz, G. Bateman et al., Phys. Plasmas 6, 1162 (1999).
${ }^{11}$ G. Bateman, A.H. Kritz, J.E. Kinsey et al., Phys. Plasmas 5, 1793 (1998).

${ }^{12}$ A.M. Dimits, G. Bateman, M.A. Beer et al., Phys. Plasmas 7, 969 (2000).

${ }^{13} \mathrm{~J}$. Weiland (private communication)

${ }^{14}$ S.M. Hamberger, B.D. Blackwell, L.E. Sharp, and D.B. Shenton, Fusion Technol. 17, 123 (1990).

${ }^{15}$ S.P. Hirshman and O. Betancourt, J. Comput. Phys. 96, 99 (1991).

${ }^{16} \mathrm{~J}$. Weiland, Collective Modes in Inhomogeneous Plasmas, Kinetic and Advanced Fluid Theory (IOP, Bristol, 2000), pp. 114, 132.

${ }^{17}$ J. Nilsson, M. Liljeström, and J. Weiland, Phys. Fluids B 2, 2568 (1990).

${ }^{18}$ J.W. Connor, R.J. Hastie, and J.B. Taylor, Proc. R. Soc. London, Ser. A 365, 1 (1979).

${ }^{19}$ A.H. Boozer, Phys. Fluids 23, 904 (1980).

${ }^{20}$ W.D. D'haeseleer, W.N.G. Hitchon, J.D. Callen et al., Flux Coordinates and Magnetic Field Structure (Springer-Verlag, Berlin, 1991).

${ }^{21}$ M. Nadeem, T. Rafiq, and M. Persson, Phys. Plasmas 8, 4375 (2001)

${ }^{22}$ M. Persson, M. Nadeem, J.L.V. Lewandowski et al., Plasma Phys. Controlled Fusion 42, 203 (2000).

${ }^{23}$ P.N. Yshmanov et al., Nucl. Fusion 30, 1999 (1990). 